\title{
1
}

\section{Performance of Passive UHF RFID Systems in Practice}

\author{
Miodrag Bolić ${ }^{1}$, Akshay Athalye ${ }^{2}$, and Tzu Hao $\mathrm{Li}^{1}$ \\ ${ }^{1}$ School of Information Technology and Engineering, University of Ottawa, Canada \\ ${ }^{2}$ Astraion LLC, NY, US
}

\subsection{Introduction}

\subsubsection{Overview}

Radio Frequency Identification (RFID) is a technology that has risen to prominence over the past decade. The clear advantages of this technology over traditional identification methods, along with mandates from supply chain giants like Wal-Mart and the Department of Defense, led to a large number of research and commercialization efforts in the early 2000s. However, almost a decade on, the early promise of widespread, ubiquitous adoption of RFID is yet to materialize. This is due to a combination of several technical and commercial factors. The technical imperfections and shortcomings existing in presentday RFID systems pose a very significant obstacle to the widespread adoption of RFID. Overcoming some of these challenges would amount to a very significant step forward towards realizing the tremendous potential of RFID technology. This book describes the ongoing efforts of some of the leading researchers in the field towards tackling the most challenging issues in today's RFID systems. With this in mind, the aim of this chapter is to clearly demonstrate, through experimentation, some of these technical challenges faced by RFID systems in practice. This chapter will enable the reader to better recognize the shortcomings of today's RFID systems and will allow for a better understanding and appreciation of the research efforts described in the rest of the book.

In this chapter, we focus on passive RFID systems operating in the Ultra High Frequency (UHF) band and adhering to the popular EPC Global Class 1 Generation 2 (Gen 2)

RFID Systems: Research Trends and Challenges Edited by Miodrag Bolić, David Simplot-Ryl, and Ivan Stojmenović

(C) 2010 John Wiley \& Sons, Ltd 
standard [1]. We begin with the characterization of a hypothetical "ideal" RFID system. We then proceed to examine the performance of practical RFID systems through simple experiments and point out the non-idealities and problems that arise in practical systems. We begin this examination by considering a simple system involving a single stationary reader and a single stationary tag in free space. We then examine systems with increasing degrees of complexity with multiple (possibly mobile) readers and tags in more challenging deployment environments. As complexity of RFID systems increase, more problems (non-idealities) are observed in the performance while problems identified with simpler systems remain. We believe that the approach of analyzing RFID systems with an increasing degree of complexity and identifying challenges as they appear will give the reader a sound understanding of the challenges facing real-world RFID systems.

Please note that this book chapter represents our viewpoint on imperfections of RFID systems. We have tried to point out some of the major issues in existing UHF RFID systems. This is not meant to be an exhaustive listing of all the possible challenges in practical UHF RFID systems, and there may be some problems and issues that have not been addressed here.

\subsubsection{Background}

RFID is a wireless technology that allows for automated remote identification of objects [2]. The major components of an RFID system are tags or transponders that are affixed to objects of interest and readers or interrogators that communicate remotely with the tags to enable identification. RFID systems exist in various flavors that can be classified based on the frequency of operation, power source of the tag and the method of communication between the reader and the tags. A detailed classification of the commercial RFID systems based on the above criteria is presented in Chapter 2. In addition, the overview of RFID technology is presented in a number of publications including [3, 4]. In this introductory chapter, we focus on passive RFID systems operating in the $860-960 \mathrm{MHz}$ band. Passive RFID tags draw the power required for operation from the radio wave transmitted to them by the reader and communicate with the reader by controlled reflection of a portion of this incident wave. This technique of communication by controlled reflection is referred to as backscatter modulation. Although this technique was used as early as World War II, RFID transponders were expensive, large devices that remained confined to military applications. However, the tremendous progress in VLSI technology along with the establishment of standards in the early 2000s, enabled RFID tags to be manufactured in high volumes resulting in a price point that initiated numerous commercial applications. The main goal of commercial RFID systems is to automate and enhance asset management by providing global asset visibility. This ability of RFID systems finds various applications in diverse fields such as supply chain management, indoor asset and personnel tracking, access control, robotics and many more.

The immense commercial potential of RFID is mainly due to the numerous advantages that the technology possesses over traditional identification mechanisms such as barcodes. Some of these advantages are: (i) passive RFID tags can be read at much greater distances than barcodes; (ii) there is no need for a line of sight between the reader and tag; (iii) multiple tags can be read at much higher rates than barcodes; (iv) RFID tags have much larger memory than barcodes which allows storage of a lot more information than just the ID; and (v) the information contained in the RFID tag can be modified dynamically using the interrogator. 
As mentioned earlier, in order to harness the advantages of RFID technology to build viable commercial solutions, a number of technical challenges needs to be overcome. Some of these challenges are common to other wireless technologies while others are unique to the RFID system to hand. Each RFID technology, including passive, semipassive and active RFID systems operating at different frequencies, poses a unique set of challenges to obtain the desired performance. In addition, design requirements, performance specifications and protocols for active, passive and semi-passive systems are also very different. Therefore, in this chapter, we will limit our discussion to long-range passive backscatter-based UHF RFID systems operating in the $860-960 \mathrm{MHz}$ band. In our opinion, this type of RFID has the most potential for significant commercial impact. As a result, it has seen the most research and standardization activity in recent years, more than other types of RFID systems. Today commercial systems of this type adhere to the EPC Global Class 1 Generation 2 (Gen 2) standard that has been in effect since 2005 [1]. Gen 2 compliant readers and tags are readily available in today's market from several vendors all over the world including Alien Technology, Impinj Inc., Motorola and others.

There have been several approaches in characterizing RFID systems. They are mainly based on (1) experimental characterization; and (2) mathematical modeling and simulation-based analysis. Experimentation is either performed in a controlled environment such as anechoic chamber, in the laboratory environment [5], [6], [7], [8] or in the application-specific setups such as conveyer applications [8]. An example of modeling and simulation-based analysis is performed in [9] where tag characteristics, propagation environment, and RFID reader parameters have been modeled and simulated. In this chapter, RFID systems are characterized through experimentation in an anechoic chamber and laboratory environment.

\subsection{Ideal RFID System}

We begin our analysis of practical RFID systems by presenting the characteristics of a hypothetical ideal RFID system. Of course, like most other ideal systems, this RFID system would be unrealizable in practice. However, formulating such a system will give us a better understanding of the problems faced by real-world RFID systems. Once again, we point out that this ideal system is formulated in the context of UHF passive RFID systems.

Since passive tags do not have a battery, they need to receive enough energy to turn on the tags' integrated circuit. Therefore, in order for a passive RFID system to operate, the tag needs to receive enough power to wake up, and its backscattered response needs to be correctly received and decoded by the reader. In addition to this basic functionality, an RFID system has several other requirements for efficient operation that will be described later as desired features. The characteristics of an ideal RFID system, that mainly correspond to the basic functionality, can be summarized as follows:

1. There exists a well-defined, controllable read zone for each reader. For every tag within its read zone, each reader has a $100 \%$ read rate or read accuracy and for tags outside its read zone, each reader has a $0 \%$ read rate. ${ }^{1}$

\footnotetext{
${ }^{1}$ For this chapter, we define read rate as the fraction of the number of times the reader is able to read a tag over the number of queries it sends to a tag. Please note that in RFID literature, the term read rate often refers to the speed at which the reader and tag communicate.
} 
2. Performance is insensitive to the physical orientation of tags.

3. Performance is insensitive to the nature of the object on which the tag is placed.

4. Performance is insensitive to the environment in which the system is deployed.

5. Multiple tags communicate with the reader in a collision-free manner and the time for reading a fixed number of tags is a deterministic function of the number of tags while utilizing the maximum allowable bandwidth.

6. Performance is unaffected by the presence of multiple readers with overlapping read zones or of multiple tags within a read zone.

7. Performance is unaffected by relative motion between the readers and tags as long as the tags remain within the read zone of the reader.

In the context of above characterization, read rate or read accuracy for each tag is defined as the percentage of times a reader is able to correctly read the tag's ID. Hence for the ideal system if a reader sends out $N$ queries to a population of tags that are all within its well-defined read zone, it receives $N$ responses from each of the tags containing their respective IDs. Similarly if a reader sends out $N$ queries to a population of tags that are all outside its read zone, it will receive no response. In the ideal system, this holds true for multiple readers with overlapping read zones, that is, if multiple readers send out $N$ queries to a population of tags that are simultaneously in the read zone of all the querying readers, then each of the readers will receive $N$ responses from each of the tags containing the respective tag IDs. As mentioned in the above characterization, the behavior of the ideal system is unaffected by factors such as orientation, environment and relative motion.

Besides these ideal characteristics, there are several other desired features that researchers are trying to bring to practical UHF RFID systems. Some of these include:

1. High level of security of an RFID system.

2. Localization of each tag within the read zone with high level of accuracy.

3. Low cost of RFID components and high return of investment.

4. Easy integration of RFID software into existing application software.

5. Simple deployment and networking of multiple readers.

6. Simple synchronization of multiple readers.

Items 4-6 are related mainly to complex RFID systems with a large number of readers.

Although the ideal RFID system is unrealizable in practice, the above characterization provides a useful reference against which to measure various performance metrics of practical systems. Moreover, one can view most of the research efforts described in the rest of the book, whether at the physical, protocol or software level, as attempts to bring practical systems as close as possible to the aforementioned ideal or desirable RFID system.

We now proceed with the analysis of practical systems by considering deployments with increasing levels of complexity, and pointing out the divergences from the ideal system that occur in practice. 


\subsection{Practical RFID Systems}

\subsubsection{Complexity of RFID Systems}

Figure 1.1 shows simple block diagrams of several RFID systems. The complexity of the systems increases from left to right. Systems consisting of stationary readers and tags are shown in Figure 1.1(a), (b), (c), while the mobile systems are shown in Figure 1.1(d), (e). Stationary readers can be attached to walls, portal constructions or ceilings resulting in fixed deployments, while mobile readers are attached to moving objects such as forklifts, hand carts or are carried by people (handheld readers) and robots. Stationary tags are attached to the objects that usually do not move during the query round, for example, tags attached to shelved items. Mobile tags are attached to mobile objects or people and they move relative to the reader antenna during the query round. Large RFID systems like the one outlined in Figure 1.1(e) require the consideration of several other issues such as networking, synchronization, data processing software and middleware in order to enable an end user to reap the benefits of a deployed RFID system.

\subsubsection{Single Reader, Single Tag}

The simplest practical RFID system from an analysis viewpoint is the one that consists of a single stationary reader and a single stationary tag (Figure 1.1(a)). Our setup for examining the performance of such a system consists of a Gen 2 compliant tag and a

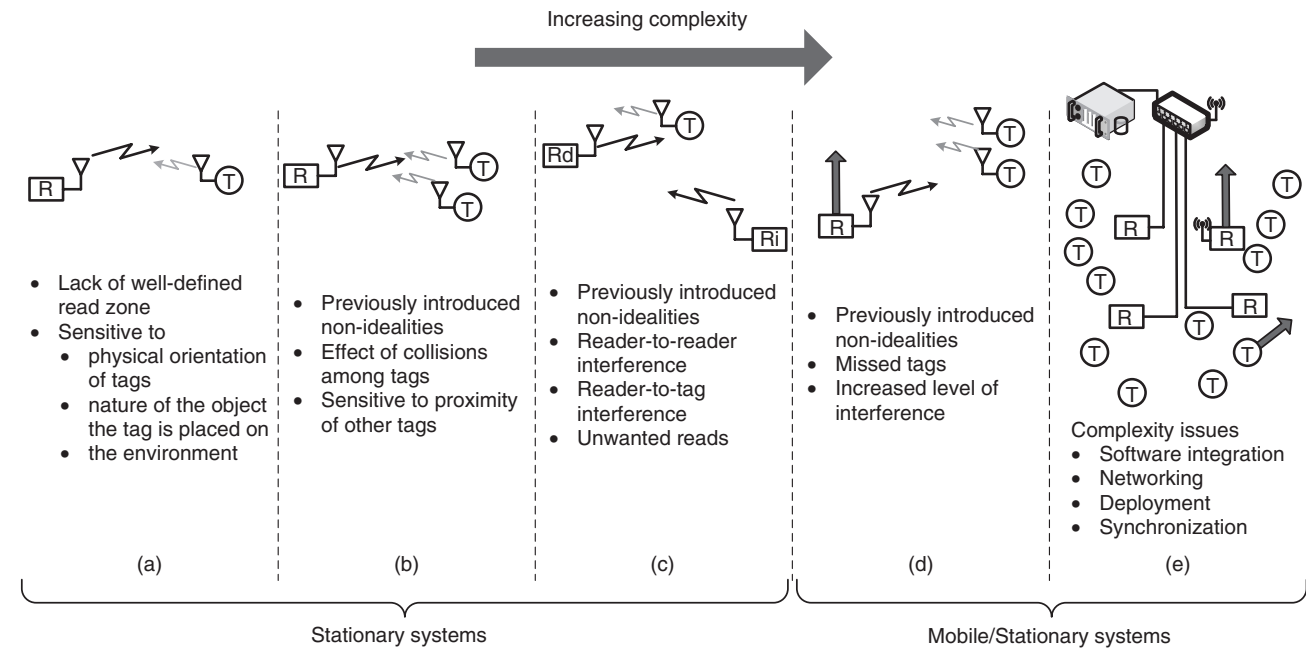

Figure 1.1 RFID systems of increasing complexity together with non-idealities encountered in each system with (a) single reader $R$ single tag $T$ system, (b) single reader multiple tag system, (c) multiple readers single tag system which includes interfering reader $R_{i}$ and reader that communicates with the tag $R_{d}$, (d) mobile reader and multiple tags, (e) complex system that includes many readers and tags where readers are connected to the router and the computer. 
Gen 2 compliant reader. The Gen 2 protocol uses a dynamic frame slotted Aloha-based anticollision protocol that enables multiple readers to communicate with a single reader. In this protocol, the reader requests tags to reply to the reader commands in defined time slots. The reader specifies a fixed number of slots in a so-called Inventory Round or Query Round. An inventory round is defined as a single cycle of an algorithm by which a reader attempts to singulate the tags within its environment. Singulation is defined as a process of identifying a single tag and reading its ID number. A Query Round begins with a Query command which species a so-called $Q$ parameter which indicates the number of slots in a query round. Each tag in a population then selects one random slot out of these slots to communicate with the reader. The reader then sends out successive Query Rep commands which designate the start of each slot. A reader may also send Query Adjust commands that dynamically increase or decrease the number of slots in the round. In its chosen slot, the tag replies with a 16-bit random number (RN16) using backscatter modulation. Upon successful reception of this RN16, the reader sends out an Acknowledge command with the same RN16 back to the tag. If this number matches the number that the tag originally sent out, the tag backscatters a Protocol Control (PC) header, followed by its EPC ID and a 16 bit CRC. A more detailed explanation of the Gen 2 standard is given in Chapter 5 . The description of the Aloha-based anticollision algorithm used is presented in Chapter 7 of this book.

In our experimental setup, the Gen 2 reader is attached to a host computer that is capable of monitoring the read rate of a detected tag, that is, the ratio of the number of times a tag responded successfully to the number of Query Rounds sent out by the reader. We are using commercial UHF Gen 2 passive dipole tag. The reader transmits at a power of $30 \mathrm{dBm}$ over a $6 \mathrm{dBil}$ gain circularly polarized antenna. In addition we deploy a sniffer device in the proximity of the tag so as to examine the actual communication happening over the wireless channel. This sniffer device is connected to an oscilloscope that stores the snapshots of the baseband signals going between the tag and the reader. Figure 1.2 shows the captured waveform for single reader, single tag scenario. Figure 1.2 shows the Query Rep commands that a reader sends out designating the tag communication slots. In its selected slot, the tag backscatters an RN16 as shown in the Figure 1.2. This is then followed by transmission of the Acknowledge command by the reader and the subsequent backscattering of the EPC ID by the tag.

Upon examination of the performance, we see that even this simple system differs from the ideal system described in Section 1.2 in several ways. The deviations are presented in Figure 1.1(a) and analyzed below.

Lack of Well-defined Read Zone: The practical single reader-single tag system does not have a specific read zone with respect to the reader antenna wherein the tag exhibits a $100 \%$ read rate and outside which the tag exhibits a $0 \%$ read rate. It has been shown in several independent works that, in the case of passive RFID systems, such as the one currently under consideration, the read range depends mostly on the power in the forward link which is needed in order to power tags IC [4, 5, 8, 9]. In free space, as a tag moves away from a reader, the mean value of the power it receives drops off as per the Friis equation describing the wireless link. As this power drops off, so does the tag's read rate until the tag reaches a place where it is unable to receive sufficient power and the read rate drops to zero. Figure 1.3 shows the read rate of a single tag as a function of the 


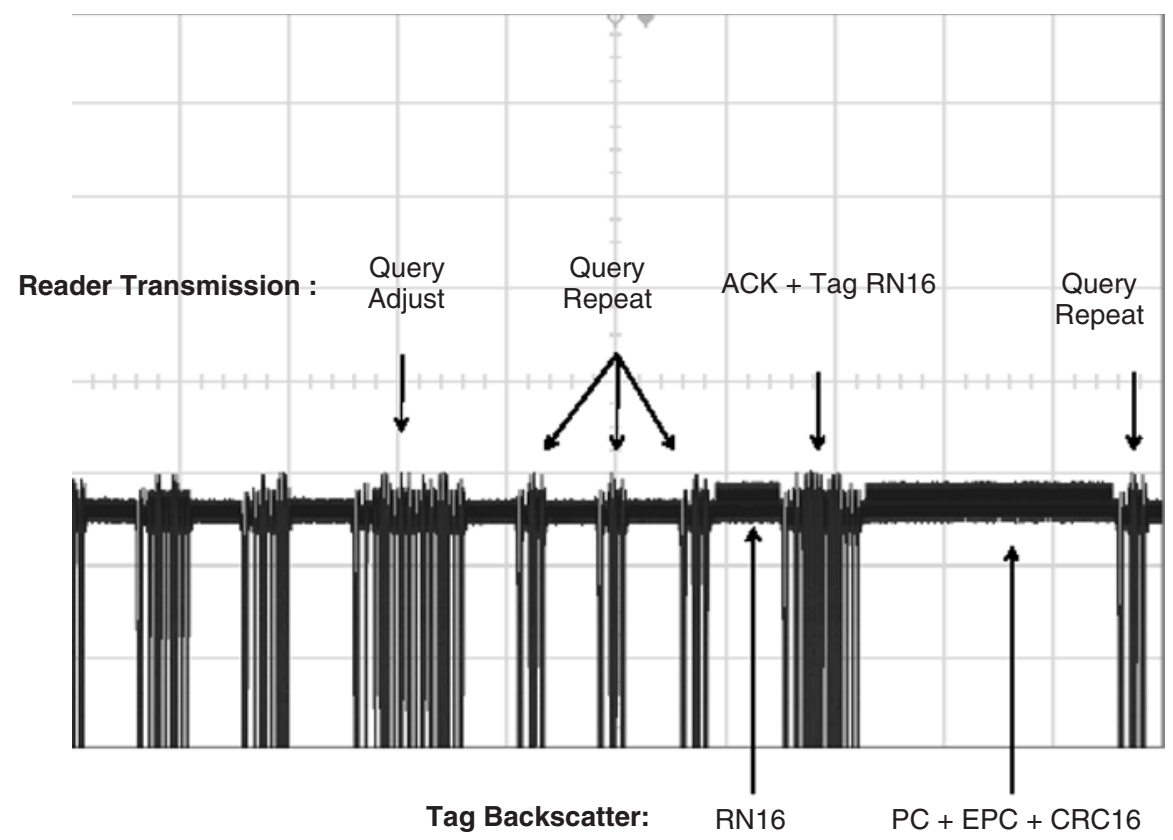

Figure 1.2 Communication between a single Gen 2 reader and single Gen 2 tag.

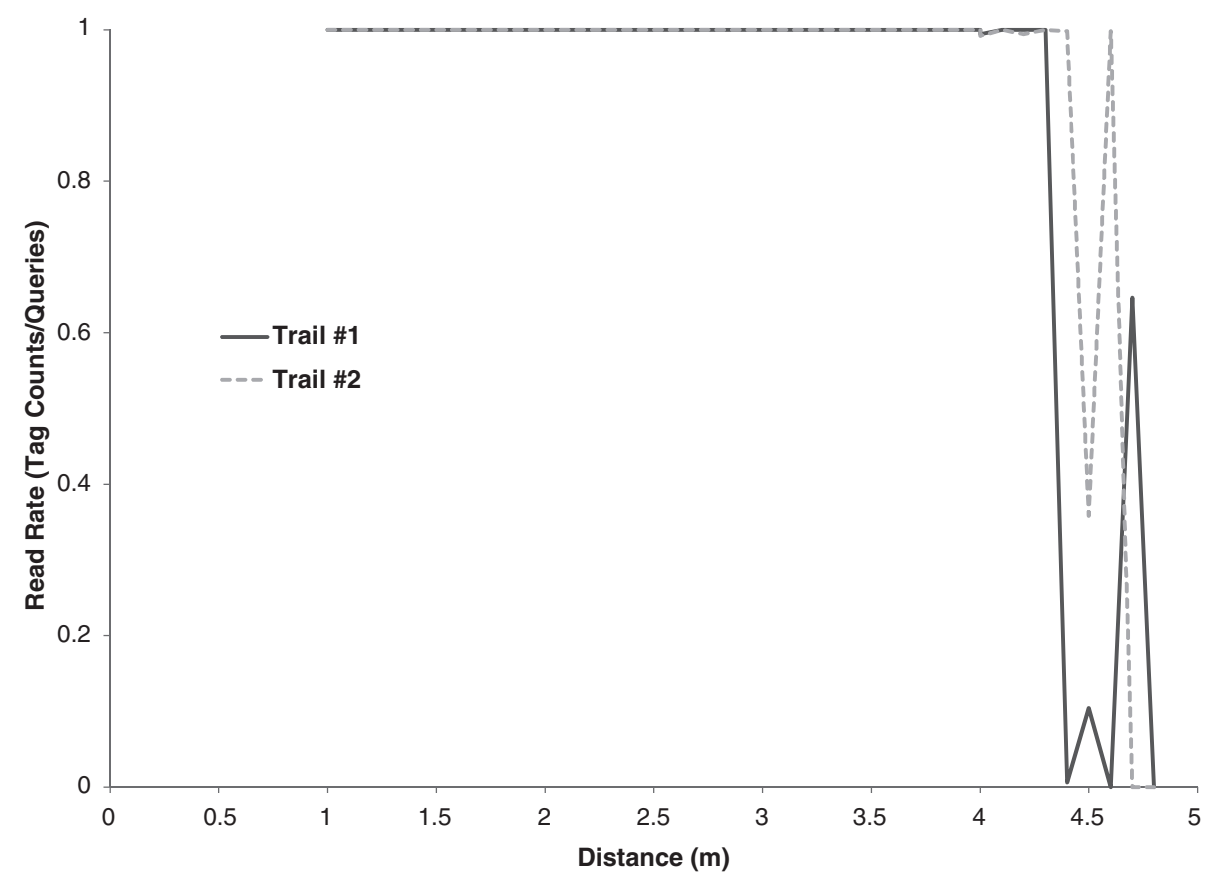

Figure 1.3 Read rate for a single tag in an anechoic chamber. 
distance from the reader. Two curves on the graph are two different trials with the same setup. The experiment is carried out in an anechoic chamber (dimensions of chamber are: length $5 \mathrm{~m}$, width $5 \mathrm{~m}$ and height $2 \mathrm{~m}$ ). For this experiment, the tag is placed in the best possible orientation with respect to the reader antenna. By performing the experiment in the anechoic chamber and by fixing the orientation of the tag and reader antennas, we wanted to avoid influence of other parameters to read rate besides the distance. The experiment was started by placing the tag at a distance of 1 meter from the reader antenna. A total of 500 Query rounds were sent by the reader, and the number of responses from the tag was noted. The steps were repeated while increasing the distance between the tag and the reader antenna in 0.5 meter steps in the range between $2 \mathrm{~m}$ and $4 \mathrm{~m}$ where the read rate was maximum, and in $0.1 \mathrm{~m}$ steps in the zone when read rates start to drop. As seen from Figure 1.3, even in an anechoic chamber, there exists a gray area around the reader antenna wherein a tag may or may not be read in a particular query round. Thus, in the practical system it is not possible to define a clear read zone as described for the ideal system. This is because of the inherent properties of electromagnetic radiation which is the basis of the communication between the reader and the tag.

Sensitivity to tag orientation: The relative orientations of the tag and the reader significantly affect the performance of a practical system. Figure 1.4 shows the effect that the tag orientation has on the read performance even when the tag is within the read range of the reader antenna. In order to collect the data we performed the experiments in an anechoic chamber with the similar setup as presented above. In this experiment,

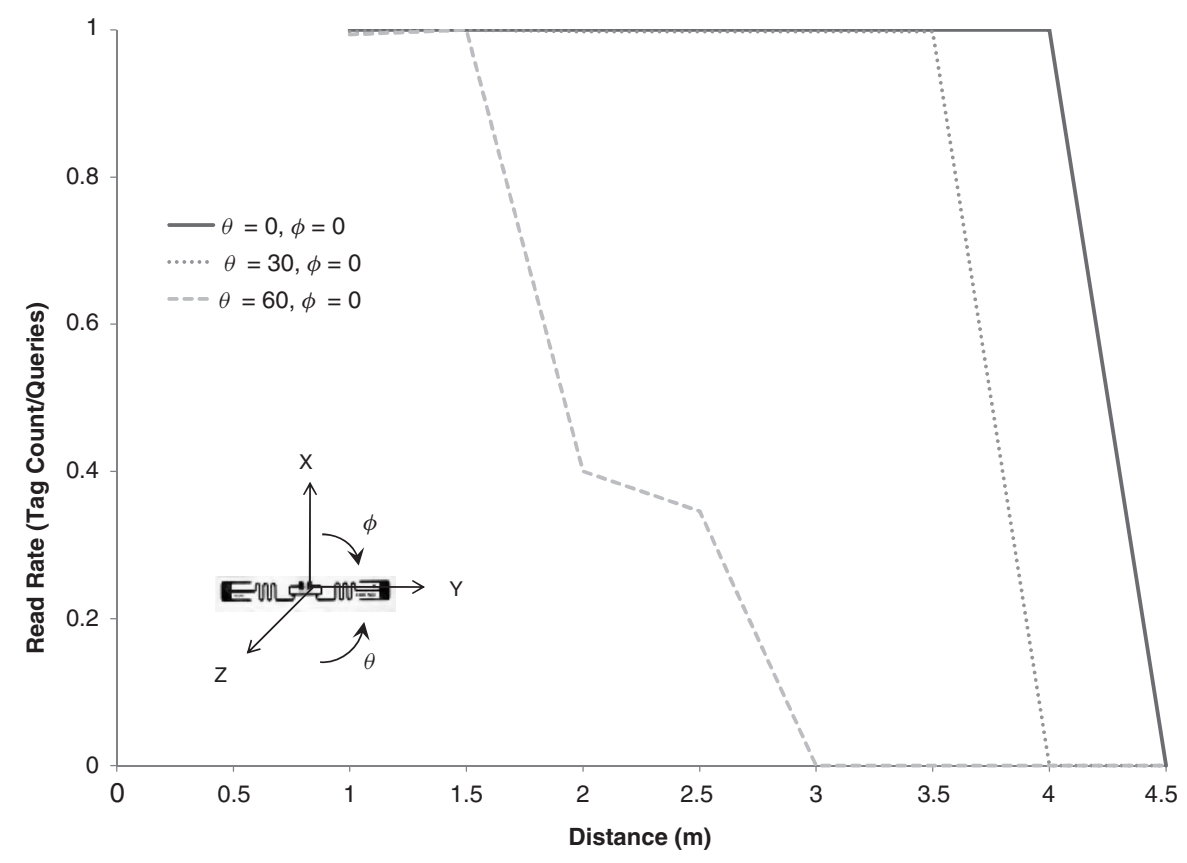

Figure 1.4 Read rate for a single tag with different orientations in an anechoic chamber. 
the reader is fixed at the position $(x, y, z)=(0,0,0)$ and tag is moved in $0.5 \mathrm{~m}$ steps in y-direction. Measurements are repeated three times: each time a tag was oriented differently relative to the orientation of the reader antenna. Read rates are recorded in the same way as in the previous experiment and presented in Figure 1.4. From Figure 1.4, it is obvious that the read range drops significantly when the orientation becomes less favorable for the tag because the tag is not able to collect enough energy to turn on the IC. In addition, Figure 1.4 demonstrates that the read rates depend on the relative orientation of the tag and reader antennas and on the distance between tag and reader.

The problem of orientation sensitivity can be handled by innovative tag antenna designs involving multiple dipoles or monopoles. In fact, ensuring orientation insensitivity is one of the most important goals in designing antennas for tags. The latest research efforts in designing efficient tag antennas are presented in Chapter 3. Experimentation with different tag orientation are published in several different works including [10]. Please note that experimentation of dependency of orientation to read rate in [10] is performed with EPCGlobal Class 1 tags (generation before Gen 2).

Sensitivity to deployment environment: The performance of a practical system is highly dependent upon the environment in which the system is deployed. Like any other wireless system, the nature of the environment affects the multipath and fading properties of the channel. This effect is even more pronounced in RFID systems due to the passive nature of the tag operation and the inherently low signal to noise ratio (SNR) of the weak backscatter signal. In order to examine the effect of environment on performance, we repeated the experiments mentioned previously in the significantly cluttered environment of a normal computer lab room (dimensions of lab room are: length $10 \mathrm{~m}$, width $6 \mathrm{~m}$ and height $4 \mathrm{~m}$ ). Figure 1.5 shows the read rate performance in the cluttered environment of a computer lab. As we can see, the deployment environment hampers the read performance of the system and also introduces some blind spots/null spots due to multipath interference and channel fading. In comparison to the read rates in the anechoic chamber, it is obvious that even for a fixed relative orientation of the reader and tags antenna it is difficult to specify the range in which the read rate is maximum.

It is clear from the above-mentioned experiments, that the read rate and read range of a tag are not solely dependent on the distance between the reader and tag, but are also affected by factors such as orientation and environment. A similar inference has been drawn in [9] and [11] wherein the authors suggested defining read range as the range in which a pre-defined read rate or accuracy of reading a tag can be achieved.

The reading accuracy problem is presented in Chapter 14 of this book together with the directions for improving reading accuracy.

Sensitivity to the nature of the object on which the tag is placed: It is known that UHF RFID systems do not perform well when attached to objects that contain metals and fluids. These materials not only attenuate the signal when placed between the tag and the reader, but also result in detuning of the tag antenna. Detuning occurs if the materials are in close proximity to the tag. The effect of metal and water on read range and read accuracy was analyzed in several publications including [5]. Read ranges were reduced up to three times in proximity of water and metal in comparison with the read range in free space. 


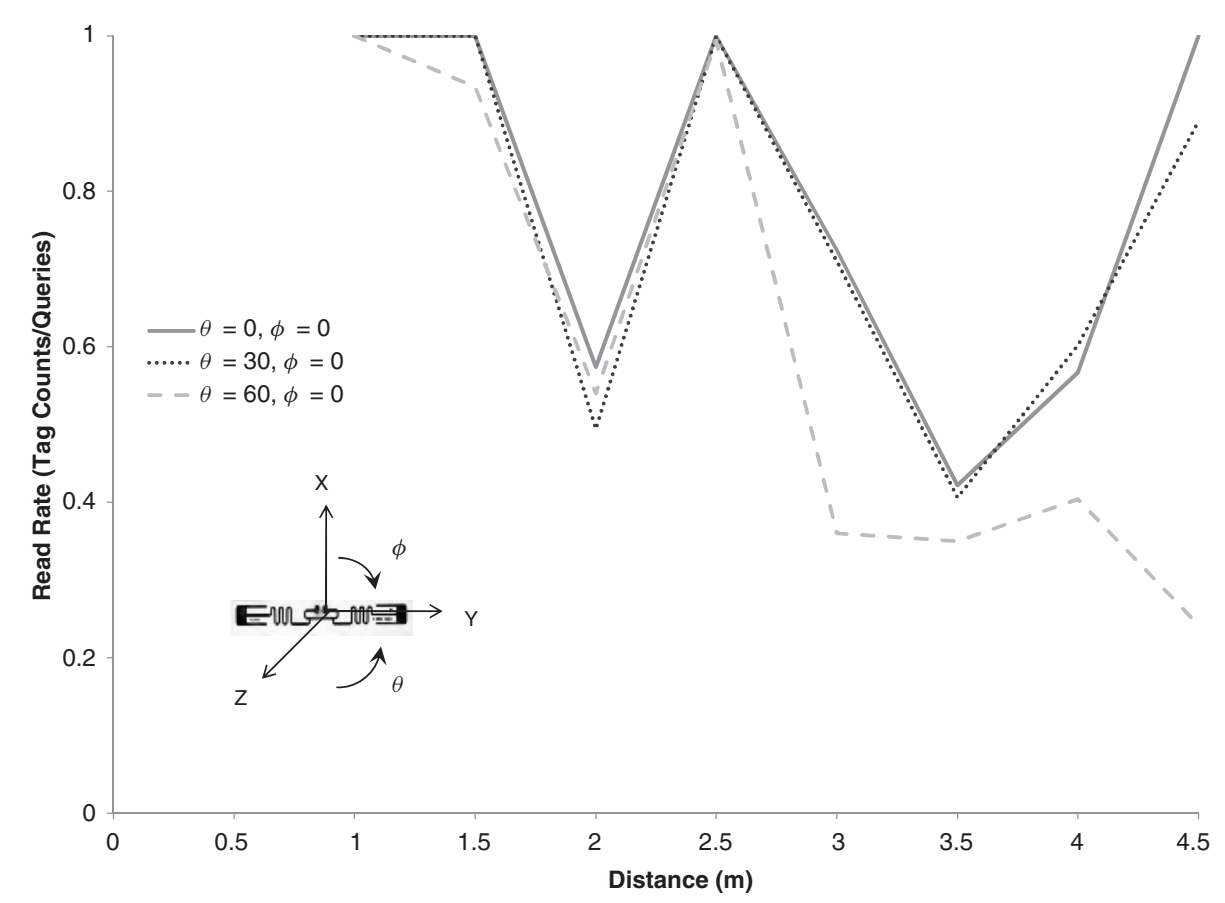

Figure 1.5 Read rate for a single tag with different orientations in a computer lab room.

Approaches to handling this problem are based on modification of tag antennas to work specifically in the proximity of metals and liquids [12]. Design of such tags is described in Chapter 3 as well.

We will now move on to more complicated practical scenarios involving multiple readers and tags. Note again, that all the non-idealities and problems identified with the simpler systems are carried forward to the more complicated systems.

\subsubsection{Single Reader, Multiple Tags}

As mentioned earlier, Gen 2 tag anticollision protocol is based on a slotted Aloha wherein multiple tags can communicate with the reader in separate time slots. The following nonidealities can be identified in the practical situations based on an examining performance.

Collision between tags: In the practical system, unlike the ideal system, collisions can occur between tags that are trying to communicate with the reader within the same query round. In the context of Gen 2, a collision occurs when two tags select the same slot number to backscatter. In this case, the reader is often unable to decipher tag transmissions and the communication attempt is unsuccessful. Figure 1.6 shows a Query round involving 2 tags where frequent collisions occur. 


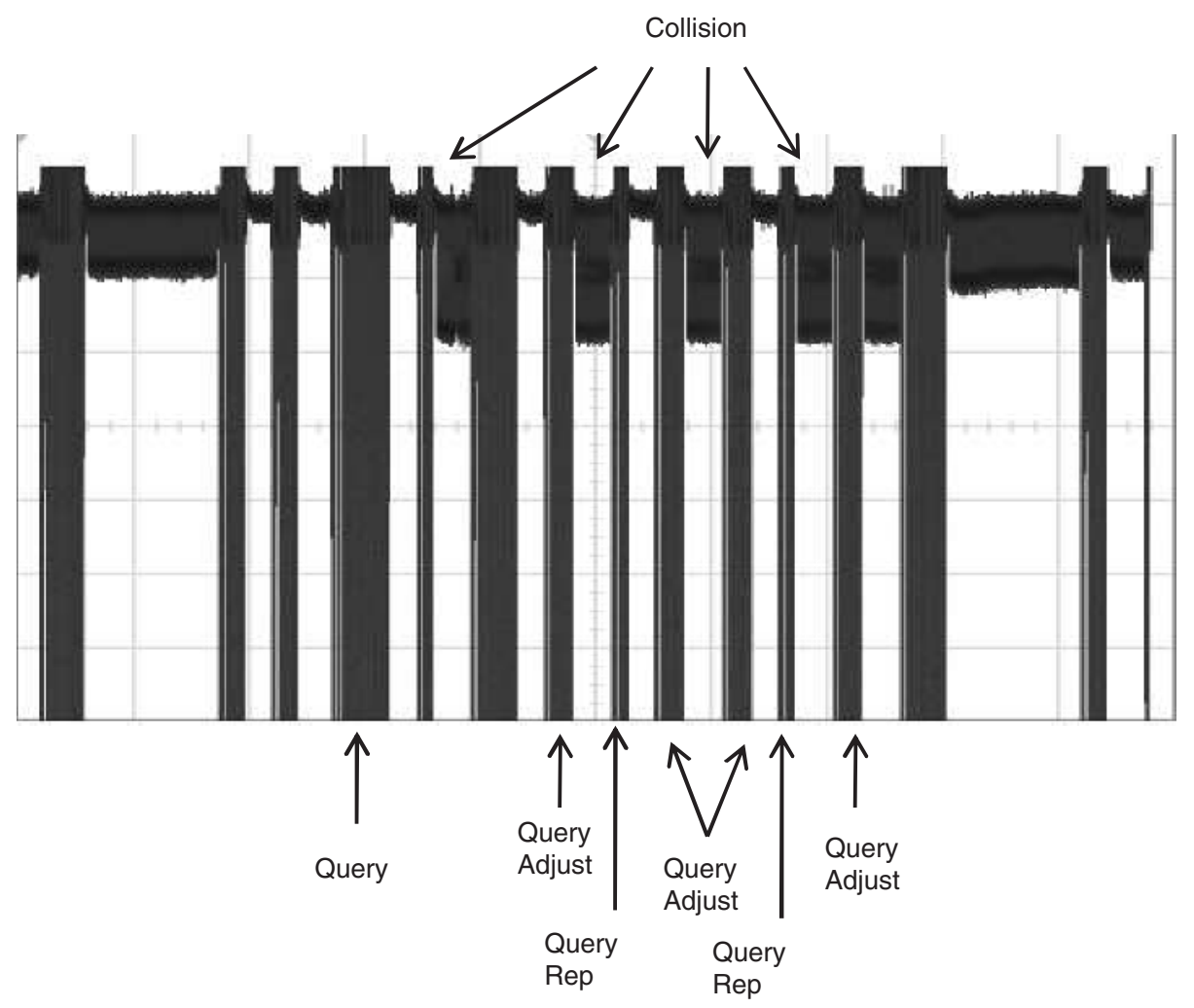

Figure 1.6 Collisions in Gen 2 query round.

Tag collision adds a significant overhead to the time needed to read a population of tags. This non-ideality is further exacerbated in scenarios involving a large number of tags simultaneously in the field of view of the reader as is the case in many commercial applications of RFID. However, these collisions are result of the air protocol selected in Gen 2 standard which is based on the Aloha paradigm. The standard does not specify implementation of the algorithm for anticollision and only gives recommendations. Chapter 7 describes different slotted Aloha anticollision algorithms with the emphasis on the methods for dynamic estimation of the number of tags to be singulated. The time to query population of tags can be minimized if the number of tags in the population is known or if it is correctly estimated.

Effect of tags in proximity to each other: In an ideal RFID system, neighboring tags will have no effect on each other's performance. In reality, however, this is not the case. In most practical applications, RFID tags are placed on objects that are densely co-located. Hence it is very important to understand the effect that the tags have on each other. The effect of proximity of the other tags to the read range and read accuracy is experimentally analyzed in [11] and [4]. 
(a) Case 1

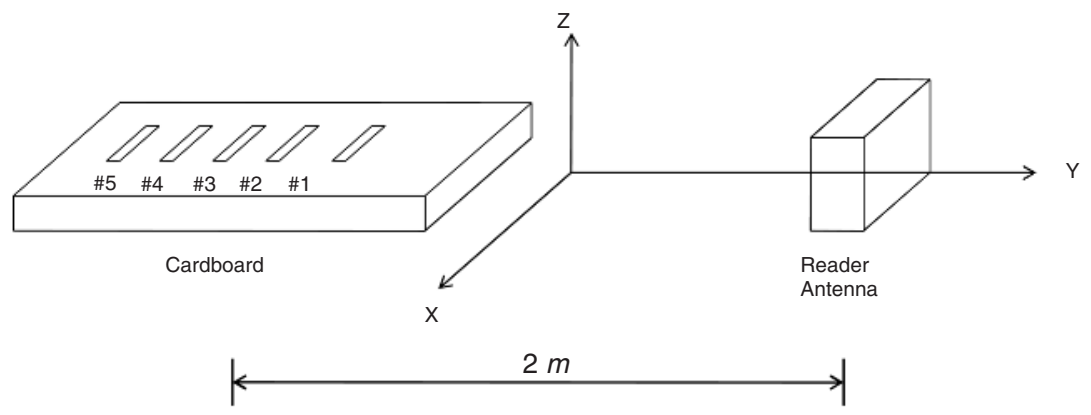

(b) Case 2
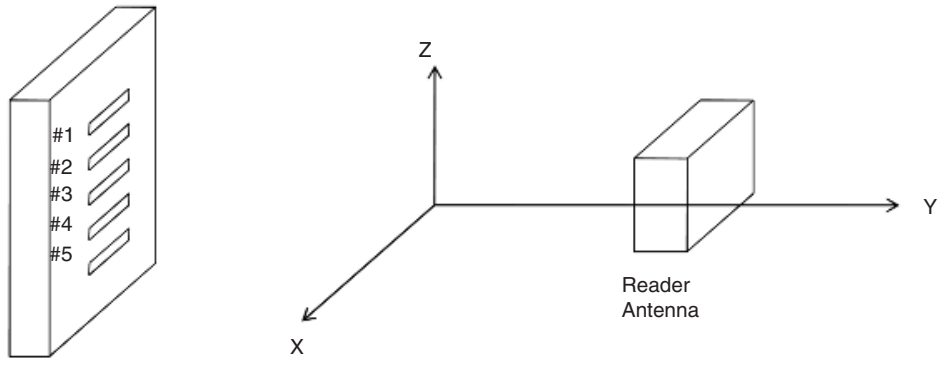

Cardboard

Figure 1.7 Experimental setup for examining effect of tag proximity on performance. Tags are spaced $1 \mathrm{~cm}$ apart and parallel to one another and (a) orthogonal to the reader antenna, (b) parallel with the reader antenna.

The presence of multiple tag antennas in close range alters the current distribution, the radiation pattern and introduces mutual impedance. As a result, tags in close proximity tend to cast a shadowing effects on the neighboring tags [4]. This shadowing effect increases as the number of tags increases and as the inter-tag distance decreases. The effect causes tags to receive less power than when no other tags are present in the proximity. This leads to a drop in the read rate or read accuracy of the tag.

In order to examine the effects of tag proximity, we use an experimental setup consisting of a Gen 2 reader with a circularly polarized patch antenna with a $6 \mathrm{dBil}$ gain and five Gen 2 tags with dipole antennas. The tags are placed $2 \mathrm{~m}$ away from reader antenna and the reader output power is set to $23 \mathrm{dBm}$. All tags are placed in the same plane on a single cardboard platform with the best possible orientation angle to the reader. This is done so as to eliminate the influence of orientation sensitivity on the measurements. The experimental setup is shown in Figure 1.7. We consider three cases; in Case 1, tags are spaced $1 \mathrm{~cm}$ apart and parallel to one another. The cardboard platform is placed along the $X-Y$ plane and the reader antenna is placed along the $X-Z$ plane. For Case 2, we place the cardboard platform along the $X-Z$ plane while keeping the position of the reader antenna unchanged. Case 3 is similar to Case 1, but we increase the inter-tag spacing to $30 \mathrm{~cm}$. The read rate results are shown in Table 1.1. Case 3 shows that when the tags 
Table 1.1 Effect of tags in proximity on tag read rate.

\begin{tabular}{lclc}
\hline $\begin{array}{l}\text { Read Rate } \\
\text { (\#reads /\#queries) }\end{array}$ & Case 1 & Case 2 & Case 3 \\
\hline Tag 1 & 0.98 & 0.45 & 0.98 \\
Tag 2 & 0.98 & 0.096 & 0.98 \\
Tag 3 & 0.56 & 0.02 & 0.97 \\
Tag 4 & 0.38 & 0.178 & 0.98 \\
Tag 5 & 0.11 & 0.87 & 0.98 \\
\hline
\end{tabular}

are spaced far enough apart, all of them have a pretty good read rate. Case 1 and Case 2 results clearly demonstrate the significant impact of tag proximity on the read rates of a tag population. This problem becomes very important in practical applications involving a large number of densely packed objects, each having a separate RFID tag.

\subsubsection{Multiple Readers, Single or Multiple Tags}

In this subsection we will consider a situation in which there are multiple stationary readers and one or more stationary tags. Having multiple readers introduces diversity and redundancy which help in solving some of the previously recognized issues. If multiple readers are used to read the same tag, the chance that the tag will be in the blind spot for all the readers is reduced. Redundant readers or one reader with multiple antennas are commonly used in industry to improve the accuracy of reading. Localization of tags might also be improved by using multiple readers. Coarse-grained localization information, based on association of tags with the reader that detected it, will be improved if tags are read by two or more readers placed at known location. The tag would then be located in the space that represents the intersection of the read ranges of these readers.

However, the presence of multiple readers in an environment gives rise to the very serious problem of interference caused by their simultaneous operation. We examine the effect of this problem on system performance below:

Interference: A system with two readers and one tag is shown in Figure 1.1(c). In this figure, communication between desired reader $R_{d}$ and tag is affected by the signal sent by the interfering reader $R_{i}$. If multiple readers attempt to singulate tags simultaneously, two types of interferences might occur: reader-to-reader and reader-to-tag interference. Readerto-reader interference or reader jamming occurs when the interfering reader affects the reception of the tag signal by the desired reader. Reader-to-tag interference or tag jamming can occur when the interfering reader affect tags reception of the signal from the desired reader.

We examine the performance of interfering readers through the following experiment. Two Gen 2 readers from different vendors were programmed to work in a dense reader mode. Dense reader mode is proposed in Gen 2 standard [1] to support large-scale enterprise applications with many readers. Spectral allocation is defined so that reader-to-reader interference is reduced. The reader transmits at a power of $30 \mathrm{dBm}$ over a $6 \mathrm{dBil}$ gain 
Table 1.2 Effect of multiple reader interference on tag read rate.

\begin{tabular}{llcc}
\hline $\begin{array}{l}\text { Reader } 1 \\
\text { Mode }\end{array}$ & \multicolumn{1}{c}{$\begin{array}{c}\text { Reader 2 } \\
\text { Mode }\end{array}$} & $\begin{array}{c}\text { Reader 1 } \\
\text { read rate }\end{array}$ & $\begin{array}{c}\text { Reader 2 } \\
\text { read rate }\end{array}$ \\
\hline $\mathrm{M}=4, \mathrm{BLF}=256 \mathrm{kHz}$ & $\mathrm{OFF}$ & 1 & - \\
$\mathrm{OFF}$ & $\mathrm{M}=4, \mathrm{BLF}=200 \mathrm{kHz}$ & - & 1 \\
$\mathrm{M}=4, \mathrm{BLF}=256$ & $\mathrm{M}=4, \mathrm{BLF}=200$ & 0.355 & 0.39 \\
$\mathrm{M}=8, \mathrm{BLF}=256$ & $\mathrm{M}=4, \mathrm{BLF}=200$ & 0.372 & 0.38 \\
$\mathrm{M}=0, \mathrm{BLF}=640$ & $\mathrm{M}=4, \mathrm{BLF}=200$ & 0.261 & 0.35 \\
\hline
\end{tabular}

circularly polarized antenna. We are using a Gen 2 dual-monopole tag that is orientation insensitive. The tag is placed in between the readers at a distance of $1.5 \mathrm{~m}$ from each reader. Read rates of each reader are recorded when the other reader is off as well as when both readers operate simultaneously. As we see from Table 1.2, the read rate of both readers dropped significantly when both of them are querying simultaneously. When both readers send their commands at same time, the tag cannot decode the collided reader signals. The more readers broadcast at same time, the harder it is to read passive tag.

There are multiple challenges in resolving interference problems that are unique to RFID systems [13]. Passive UHF tags have limited tuning capacity and their reception will be affected by signals from multiple readers even if the readers operate at different frequency channels. In addition, readers transfer high amounts of power that is needed to power up the tag. This high power worsens the interference problems.

Reader-to-reader interference problem is addressed in the Gen 2 standard by introducing the dense reader mode. In this mode, lower data rates are specified, and Miller Subcarrier encoding is used so that the channel for reader and tag transmission can be well defined and separated. Dense reader mode is also analyzed in Chapter 5 of this book. For regions with only narrow frequency band available in UHF band such as Europe, the listen before talk approach together with applying spectral mask constraints are viable solutions $[1,14,15]$.

Even though readers transmit in separate frequency channels, they can still cause readerto-tag interference. In [16], it has been shown that the tag might function even if there are interfering readers. If desired and interfering readers transmit simultaneously, tags will be able to detect the signal from the desired reader if that signal is much stronger than the signal from the interfering reader. Experimentation was performed at the frequency of $866 \mathrm{MHz}$. Tags can detect the signal from the desired reader if the following conditions are met: (1) the signal power from the desired reader needs to be $6 \mathrm{~dB}$ higher than the signal power from the interfering reader when the difference in reader's carrier frequencies is more than $800 \mathrm{kHz}$; and (2) the signal power from the desired reader needs to be $13-20 \mathrm{~dB}$ higher than the signal power from the interfering reader when the difference in readers carrier frequencies is less than $800 \mathrm{kHz}$ [16].

\subsubsection{Mobile Readers and/or Mobile Tags}

Both reader and tags can be mobile. Figure 1.1(d) shows an example in which the reader is mobile and the tags are stationary. This corresponds, for example, to the situation when 
the reader is attached to a forklift and the tags are placed on items that are on the shelves. In mobile systems, it will not happen that the tag remains in the blind spot. Due to the mobility of tags and/or readers, the reader will eventually be able to read the tag. While mobility can aid in handling some of the identified issues, it also introduces new problems into the system. Centralized algorithms for reducing interference might fail if readers are mobile and some tags might not be read because they appear in front of the reader for the limited amount of time.

Missed tags: Let us consider the case in which tags are mobile and the reader is stationary. As pointed out in [17], RFID tag antennas designed for stationary applications operate reliably when tags move at low speed. For example, a speed of about $16 \mathrm{~km} / \mathrm{h}$ is considered low enough for reliable operation. In [18] the effect of accuracy is examined when ten single-dipole tags are moved at the speed of $1 \mathrm{~m} / \mathrm{s}$ and were kept at $1 \mathrm{~m}$ from the reader. Different relative orientations between tags are explored. It was determined that the minimum distance between tags should be $4 \mathrm{~cm}$ in order to be able to detect all the tags. Other experimentation is described in [19], where an RFID reader is placed on the side of the conveyer belt whose speed is $2 \mathrm{~m} / \mathrm{s}$ and tags are attached on boxes placed on the conveyer belt. The system is first calibrated to avoid unwanted reads. Excellent read rate is achieved even in cases when 50 tags are placed on the same box and the speed of the conveyer belt is $2 \mathrm{~m} / \mathrm{s}$ and when the reader is $44 \mathrm{~cm}$ away from the tags. However, the high reading rate in both papers might be due to the fact that the reader was placed very close to the tags.

Simulations with detection of high speed objects are described in Chapter 9 of this book. It has been shown that it is possible to read four tags at a distance of $4 \mathrm{~m}$ at speeds of $250 \mathrm{~km} / \mathrm{h}$ with appropriate setting of parameters of Gen 2 protocol. It also shows that much better performance for high speed tags can be achieved using tag talk first protocols. The experimentation with mobile tags attached to a vehicle is performed in [20]. In the experiment, six tags are attached to the windshield and two antenna are placed at the height of $4.2 \mathrm{~m}$ with an angle of 45 degrees. High read rate is achieved at the speed of $10 \mathrm{~km} / \mathrm{h}$, while very low read rates are achieved for speeds above $70 \mathrm{~km} / \mathrm{h}$. The method for predicting the read rate is presented in [20] and it relies on the support vector machine model.

Mobile readers are used in combination with stationary tags in several different applications for localization, mapping and navigation of robots and people. In almost all applications stationary tags are used as landmark tags to assist in positioning of the mobile readers and their carriers. In [21] it was also confirmed that the major problem is how one can cope with low reading accuracy. If the landmark tag is not read, the position cannot be determined. One possible solution, self-localization of robots that takes into account unreliable RFID readings, is proposed in [21]. There, the robot first collects information about the read landmark tags and the number of their detection during the training phase. This information is stored and used to compare with the real measurement results during operation of the robot.

\subsubsection{Large Deployments Including Many Readers and Tags}

Figure 1.1(e) shows an RFID system consisting of a large number of readers and tags. The figure shows RFID system with four readers of which three are stationary and connected 
using Ethernet to the switch. One reader is mobile and it communicates with the switch using a wireless link. The switch is connected to the host that runs middleware software. The role of the middleware is to aggregate the data from the deployed readers, filter and process it as per case-specific requirements and present it to the higher level software. In addition to the physical non-idealities that we have described, this large deployment of multiple readers and tags gives rise to a whole new set of challenges related to the middleware, application software and network management.

Synchronization of many readers: In large deployments, implementing the synchronization scheme is a significant challenge. Many algorithms have been developed to reduce interference in large-scale deployments (Chapter 11 of this book). Centralized synchronization is impractical for large-scale solutions and should be avoided [22]. In addition, in multivendor environments, such as shopping centers, receiving dock doors might belong to different organizations and synchronization is again impractical [15]. In these situations, readers will need to be able to operate asynchronously of each other and be truly event driven [15].

Easy integration of RFID software into existing application software: Full benefit of an RFID system can only be utilized if data collected from the RFID system is used for decision-making [23]. Therefore, the RFID reader system needs to be integrated into the existing application software. RFID middleware software is used to provide, besides other functions, easy integration with legacy applications. If the applications have proprietary interface, then the integration becomes complicated [23]. RFID Middleware as a part of EPCGlobal architecture is described in Chapter 6. The architecture of RFID system is presented in Chapter 10.

Installation and tuning: In deployments with a large number of readers, installation of the RFID components can be complicated. It requires installation of power connections and network cabling. In addition, readers and antennas have to deployed properly to reduce interference among the readers and other wireless devices or machineries to the RFID networks. Improper deployment can result in some areas being not properly covered by RFID readers. Therefore, the RFID system needs to be tuned by the experienced hardware engineer [23] to assure that required performance is achieved.

\subsubsection{Other Desired Features of Practical RFID Systems}

As mentioned earlier, in addition to the basic operational characteristics embodied by the ideal RFID system, practical systems have some other features that are necessary in enabling commercial applications. We describe a couple of these below.

Privacy and security in Gen 2: Privacy and security aspects are extremely important for successful deployment and application of RFID systems. A large number of publications have recently appeared that deal with improving or developing security and authentication frameworks for RFID applications [24].

RFID tags based on Gen 2 standard are considered to belong to low-end systems based on their capacity to implement schemes for security. Current security and privacy 
mechanisms in the Gen 2 standard, although better than previous standards, are still considered weak (Chapter 16 of this book). In the Gen 2 standard, the reader does not transmit the EPC code, parts of the tag memory can be locked, a kill command can permanently disable the tag. Functions such as access to special memory, ability to modify tags and the ability to kill tags require a 32-bit access password. However, the 32-bit access password can be eavesdropped and then easily computed. More advanced security and privacy methods are described in Chapters 16 and 17 of this book. As is pointed out in Chapter 2 of this book, adding security features to passive tags would increase the complexity of the tag's hardware and then likely reduce the tag's range and throughput performance. This tradeoff makes the task of designing security mechanisms for passive RFID systems all the more challenging.

Reducing ambiguity in tag location: Unlike an ideal system, the practical reader-tag RFID system is unable to determine the precise location of the tag within the read zone of the reader. As mentioned earlier, one of the main goals of an RFID system is to provide global asset visibility. In most practical applications, precise asset location is an important attribute of asset visibility in order to automate the asset management process. The application of traditional ranging techniques such as those based on received signal strength (RSS), time of arrivals (TOA), time difference of arrivals (TDOA) and phase of arrivals (POA) to passive RFID is very challenging due to the weak backscatter signal used to communicate back to the reader. Localization techniques such as trilateration rely on precision of ranging techniques. This is the reason why many RFID localization solutions are based on using landmark tags or some kind of map matching. Precise localization using passive RFID is an important problem that is the focus of a number of research efforts today. The overview of localization algorithms is presented in Chapter 2 and detailed analysis of algorithms for ranging and localization is presented in Chapter 15 .

Reducing the unwanted reads: The fact that the reading zone is not properly defined causes tags to be read by multiple readers. In some applications, this is not desired. For example, in dock door application, each reader covers a different door. It is important to detect which door the pallet went through. If the tags on the pallets are read by multiple readers, this will introduce ambiguity in associating pallets with corresponding dock doors. Reading of the tags by the readers that cover neighboring doors are called unwanted reads. Other names used in practice are unintended reads and cross reads [25]. Solutions against unwanted reads are based on both hardware (shielding) and software (filtering of data).

\subsection{Overview of the Book}

This chapter presented problems and non-idealities in current passive UHF RFID systems. The rest of the book describes approaches to deal with the defined problems. In addition, the book tackles a number of other issues that have not been described in this chapter. Therefore, we summarize major problems, methods and approaches covered in the book.

Chapters 3-5 deal with design of tag antennas, tag and reader circuit for passive RFID systems operating in UHF band. Chapter 3 introduces the design of antennas for passive UHF tags. The chapter also describes the effects of environmental factors including proximity of dielectric materials and metals on performance of the antennas. Then, several 
approaches for designing antennas that operate in proximity of metals have been proposed. In Chapter 4, tag's architecture is presented. RF, baseband and memory design are detailed. Low power design issues are pointed out. Chapter 5 focuses on the reader design issues that are unique to RFID systems including handling transmitter leakage during tag reception, frequency generation, transmit linearity and transmit AM noise.

Chapters 6 and 10 cover EPCGlobal architecture framework and protocols. Chapter 10 analyzes services that the RFID system supports and defines the system architectures. The chapter then analyzes a number of EPCGlobal specifications that standardize interfaces to configuration, monitoring and data processing services. Chapter 6 describes RFID middleware in details. It focuses on the following aspects: reader management, data management and storing and sharing data in RFID networks.

Non-idealities of the RFID systems are tackled throughout the book. The problem of read range is considered in Chapters 2 and 14. The parameters that affect read range in a RFID system are pointed out and ways to achieve longer reading ranges are introduced. Parameters that affect the throughput of RFID systems are considered in Chapter 2. Reading accuracy is explained in more detail in Chapter 14.

Localization of passive and active tags is the application that is attracting increasing interest. The localization problem is introduced in Chapter 2 and elaborated in Chapter 15.

Privacy and security aspects of RFID networks are considered in Chapters 16 and 17. Chapter 16 focuses on low level approaches and covers attacks against common RFID components and lightweight cryptography. Chapter 17 describes higher level aspects of security and privacy including description of the cryptographic protocols for privacy, identification with privacy, and authentication without privacy.

Even though the book mainly deals with passive RFID system, active RFID systems are also described in Chapter 2. Some localization solutions based on active RFID systems are mentioned in Chapter 15. Dual frequency active RFID systems are presented in Chapter 18. Chapter 18 is dedicated to novel technologies and it is especially focused on energy harvesting. Energy harvesting is also briefly discussed in Chapter 19.

In Chapter 19, a methodology that allows translation of the models of RFID system automatically onto a hardware platform to enable real-time verification in the target operating environment is presented. A detailed description of the simulator and the emulator is provided.

Tag identification protocols are presented in Chapters 7, 8 and 9. Reader talk first protocols including Aloha and tree-based protocols are described in Chapters 7 and 8 respectively, while tag-talk-first approach is described in Chapter 9. Combinations of tree-based and Aloha-based algorithms are described in Chapter 7. In general, the number of tags to be singulated is not known. For both tree- and Aloha-based protocol, knowledge of the number of tags would reduce the average time it takes for the protocol to singulate all the tags. Therefore, in Chapters 7 and 8 ways to estimate number of tags while the algorithm is running are described. Tag talk first protocols are analyzed in Chapter 9. Detailed comparisons with Aloha-based protocols have been performed regarding throughput and speed of reading.

In the near future, RFID deployments with large number of readers will be common. In these applications, reducing interference among the readers and performing synchronization of the readers will become a major problem. Detailed comparison of reader anticollision algorithms is presented in Chapter 11. Extension of the tree based protocols 
for multiple collaborative readers is described in Chapter 8. An example of the reader anticollision algorithm is also presented in Chapter 12.

With improving technology and dropping price of RFID readers, dense reader deployments will soon become reality. In addition to improving accuracy, densely deployed readers can be used, for example, to estimate more accurately the location of the tagged items. On the other hand, in order to reduce overall power consumption in the network and reduce amount of interference, it is important to turn off redundant readers when they are not needed. A redundant reader can be safely turned off or removed from the network without affecting the number of tags covered. Detecting redundant readers is a complex problem that is described in Chapter 12.

Several other networking problems are considered in the book. They include a problem when readers that are connected wirelessly and communicate using multi-hop communication need to optimally report detected tags. The solution to eliminate redundant tag reports generated by multiple readers is provided in Chapter 12. Delay-tolerant mobile networks of RFID readers are introduced in Chapter 13. In these networks information among stationary readers is transferred using mobile tags. The many challenges that this type of network introduces are presented in Chapter 13.

\subsection{Conclusion}

Although radio frequency identification is a rapidly emerging technology, several technical challenges need be to overcome to enable its ubiquitous adoption. In this chapter, we have examined some of these important technical challenges in present-day UHF passive RFID systems. We did this by formulating a hypothetical ideal RFID system which exhibits optimal performance in various aspects. We then examined the performance characteristics of practical RFID systems and how they diverge from the ideal system. Overcoming these non-idealities will be the key in enabling RFID technology to achieve its immense potential. We hope that this chapter has given the reader a better understanding of what ails the RFID systems of today. With this understanding, the reader can better appreciate the research efforts being described in the rest of the book and how each of these efforts fits into the bigger picture of enabling ubiquitous adoption of UHF RFID systems.

\section{Acknowledgements}

We would like to thank Alexey Borisenko for performing some of the experiments. We would also like to thank Dr. Michael Knox and Dr. Mustapha Yagoub for giving constructive comments that helped improve this manuscript.

\section{References}

[1] EPCglobal Inc. (2008) EPC ${ }^{\mathrm{TM}}$ Radio Frequency Identification protocols class-1 generation-2 UHF RFID protocol for communications at $860 \mathrm{mhz}-960 \mathrm{mhz}$, Standard Specification version 1.2.0.

[2] Wyld, D. C. RFID (2005): The right frequency for the government. A research monograph from the IBM Center for the Business of Government.

[3] Want, R. (2006) An introduction to RFID technology, IEEE Pervasive Computing, 5(9): 25-33.

[4] Dobkin, D. M. (2007) The RF in RFID: Passive UHF RFID in Practice. Oxford: Elsevier-Newnes. 
[5] Derbek, V., Steger, C. Weiss, R. Preishuber-Pflugl, J. and Pistauer, M. (2007) A UHF RFID measurement and evaluation test system, Electrotechnic and Informationstechnik, 124(11), 384-390.

[6] Buettner, M. and Wetherall, D. (2008) "An emperical study of UHF RFID systems," in MobiCom, San Francisco, California, USA.

[7] Muhlmann, U. and Witschnig, H. (2007) "Hard to read tags": an application-specific experimental study in passive UHF RFID systems, Electrotechnic and Informationstechnik, 124(11). 391-396.

[8] Ramakrishnan, K. N. (2005) Performance benchmarks for passive UHF RFID tags, M.S. thesis, University of Kansas.

[9] Nikitin, P. and Rao, V. (2006), Performance limitations of UHF RFID systems, in IEEE Antennas and Propagation Symposium, pp. 1011-1014.

[10] D'Mello, S., Mathews, E., McCauley, L. and Markham, J. (2008) Impact of position and orientation of RFID tags on real time asset tracking in a supply chain, Journal of Theoretical and Applied Electronic Commerce Research, 3(1), 1-12.

[11] Currie, I. A. and Marina, M. K. (2008) Experimental evaluation of read performance for RFID-based mobile sensor data gathering applications, in Proceedings of the 7th International Conference on Mobile and Ubiquitous Multimedia, Umea, Sweden, pp. 92-95.

[12] Mitsugi, J. and Hada, H. (2006) Experimental study on UHF passive RFID readibility degradation, in Proceedings of the International Symposium on Applications and the Internet.

[13] Impinj. Inc, (2007) RFID communication and interference, White Paper, Grand Prix Application Series.

[14] Leong, K. S., Ng, M. L. and Cole, P. H. (2006) Positioning analysis of multiple antennas in dense reader environment, in Applications and the Internet Workshops.

[15] Tuner, C. The dense reader problem in Europe, White Paper, November 2005.

[16] Martinez, R. Interference in RFID systems, Presentation for SG3.

[17] Rao, K. V. S., Nikitin, P. V. and Lam, S. F. M. Antenna design for UHF RFID tags: A review and a practical application, IEEE Transactions on Antennas and Propagation, 53(12).

[18] Rahmati, A., Zhong, L., Hiltunen, M. and Jana, R. (2005) Reliability techniques for RFID-based object tracking applications, in 37th Annual IEEE/IFIP International Conference on Dependable Systems and Networks, pp. 113-118.

[19] Ren, Z., Tan, C. C., Wang, D. and Li, Q. (2009) Experimental study of mobile RFID performance, in International Conference on Wireless Algorithms Systems and Applications, Boston, MA, pp. 12-20.

[20] Jo, M., Youn, H. Y., Cha, S.-H. and Choo, H. (2009) Mobile RFID tag detection influence factors and prediction of tag detectability, IEEE Sensor Journal, 9(2): 112-119.

[21] Schneegans, S. Vorst, P. and Zell, A. (2007) Using RFID snapshots for mobile robot self-localization, in Proceedings of the 3rd European Conference on Mobile Robots (ECMR 2007), Freiburg, Germany, pp. 241-246.

[22] Tanaka, T. and Sasase, I. (2007) Interference avoidance algorithms for passive RFID systems using contention-based transmit abortion, IEICE Transactions on Communications, E90-B(11): 3170-3180.

[23] Bhuptani, M. and Moradpour, S. (2005) RFID Field Guide: Deploying Radio Frequency Identification Systems, Sun Microsystems Press, Prentice Hall.

[24] Ahson, S. A. and Ilyas, M. Eds., (2008) RFID Handbook: Applications, Technology, Security and Privacy. Boca Raton, FL: CRC Press.

[25] Krishna, P. and Husalc, D. (2007) RFID infrastructure, IEEE Communications Magazine, 45(9): 4-10. 